\title{
ACOUSTICS2008/2813 \\ Sensitivity analysis and non linear parameters estimation of porous materials from normal sound coefficient absorption measurements
}

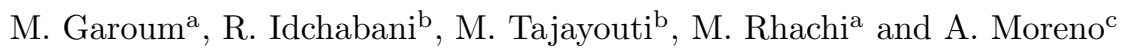 \\ ${ }^{a}$ Ecole Supérieure de Technologie de Salé, Laboratoire Energétique Matériaux et Environnement, Avenue du \\ Prince Héritier, BP 227, 10000 Salé, Morocco

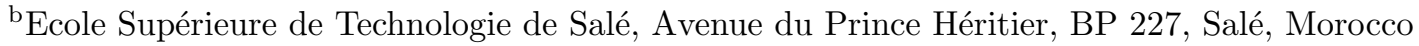 \\ ${ }^{\mathrm{c}}$ Instituto de Acústica, CSIC, 144 Callé Serrano, 28006 Madrid, Spain
}

In literature, various models have been proposed in order to predict characteristic impedance Zc and wave number kc of porous materials. To use these models their involved nonacoustical parameters (i. e. flow resistivity, tortuosity porosity...) are usually measured. Unfortunately measurements require specialised equipments and are often difficult on loose porous materials leading most often to erroneous values. Moreover, the inverse estimation of theses parameters from experimental data is not an easy task as believed. This is due to the non linearity of models and the presence of some parameters with weak and/or linearly dependent influences. In this work an approach based on the tradeoff between magnitude and linear independence of the sensitivity matrix components is presented in order to achieve an efficient ranking of parameters according to their inherent ease of estimation. Next this approach is associated to Genetic Algorithms to minimize the least squares norm between measured and modelled normal sound absorption coefficient. For five widely used models in literatures, the application of the proposed inverse estimation procedure on loose granular cork and vegetable fibres, shows that, unlike classical gradient approaches methods, better estimation has been obtained. 